\title{
Binding and interlayer force in the near-contact region of two graphite slabs: experiment and theory
}

\author{
Tim Gould, ${ }^{1, \text { a) }}$ Ze Liu, ${ }^{2}$ Jefferson Zhe Liu, ${ }^{3, \text { b) }}$ John F. Dobson, ${ }^{1}$ Quanshui Zheng, ${ }^{2,4}$ and S. Lebègue ${ }^{5}$ \\ 1) Queensland Micro and Nano Technology Centre, Nathan campus, Griffith University, 170 Kessels Road, Nathan, \\ QLD 4111, Australia \\ ${ }^{2)}$ Department of Engineering Mechanics and Center for Nano and Micro Mechanics, Tsinghua University, \\ Beijing 100084, China \\ 3) Department of Mechanical and Aerospace Engineering, Monash University, Clayton, VIC 3800, \\ Australia \\ 4) Institute of Advanced Study, Nanchang University, Nanchang, China \\ ${ }^{5)}$ Laboratoire de Cristallographie, Résonance Magnétique et Modélisations (CRM2, UMR CNRS 7036) Institut \\ Jean Barriol, Université de Lorraine BP 239, Boulevard des Aiguillettes 54506 Vandoeuvre-lès-Nancy, \\ France
}

Via a novel experiment, Liu et al. [Phys. Rev. B, 85, 205418 (2012)] estimated the graphite binding energy, specifically the cleavage energy, an important physical property of bulk graphite. We re-examine the data analysis and note that within the standard Lennard-Jones model employed, there are difficulties in achieving internal consistency in the reproduction of the graphite elastic properties. By employing similar models which guarantee consistency with the elastic constant, we find a wide range of model dependent binding energy values from the same experimental data. We attribute some of the difficulty in the determination of the binding energy to: i) limited theoretical understanding of the van der Waals dispersion of graphite cleavage, ii) the mis-match between the strong bending stiffness of the graphite- $\mathrm{SiO}_{2}$ cantilever and the weak asymptotic inter-layer forces that are integrated over to produce the binding energy. We find, however, that the data does support determination of a maximum inter-layer force that is relatively model independent. We conclude that the peak force per unit area is $1.1 \pm 0.15 \mathrm{GPa}$ for cleavage, and occurs at an inter-layer spacing of $0.377 \pm 0.013 \mathrm{~nm}$.

\section{INTRODUCTION}

Graphene has attracted sustained interest in recent years because of its unusual electronic, magnetic and mechanical properties $^{1-5}$. Applications that depend on mechanical properties include, for example, flexible touchscreens ${ }^{6}$ and graphene-coated oscillating sensor devices. These can be based on large-scale high-quality flexible vapour deposited graphene sheets ${ }^{7}$. To model such applications one requires a reliable knowledge of the force and binding energy between graphene layers, quantities that have recently been controversial both at the theoretical and experimental level. For example, results for the graphene layer binding energy vary by at least a factor of two between different experiments ${ }^{8-10}$. An equally large spread of predictions is found amongst theoretical analyses $^{11-18}$. Any fresh experimental insight on this system is therefore important.

A recent paper ${ }^{19}$ from some of the authors used the bulk mechanical properties of graphite to establish a value for the inter-graphene-layer binding energy indirectly from displacement measurements. In the experiment, a flake of graphite with a $\mathrm{SiO}_{2}$ backing was allowed to cantilever into a stepped graphite substrate and atomic force microscopy (AFM) used to measure the profile of the cantilever. This profile was then fit to the

\footnotetext{
a) Electronic mail: t.gould@griffith.edu.au

b) Electronic mail: zhe.liu@monash.edu
}

prediction from a finite element analysis using a binding force of cleavage vs. displacement relationship derived from a parameterised Lennard-Jones (LJ) potential. One parameter (the effective binding energy) was allowed to vary in the LJ potential and a best fit was found to the measured data predicting a binding energy of $0.19 \pm 0.01 \mathrm{~J} / \mathrm{m}^{2}$.

In this paper we shall first discuss certain problems in the previous theoretical analysis of experiment ${ }^{19}$. The most significant of these is an internal inconsistency between the effective elastic coefficient used in Hooke's law for the contact layer $(20.0 \mathrm{GPa})$, and other layers (36.5GPa), discussed in depth in Section IIB. We will show that this discrepancy comes from limitations of the popular Lennard-Jones model when applied to layered materials, and that layered materials present a particularly difficult case for indirect measurements of energy. This difficulty is due to a combination of poorly understood theory, and variable length and force scales for different energetic contributions.

We will then reanalyse the experimental data from Ref. 19 using improved binding energy models to better estimate the energetics of cleavage. These improved models remove the discrepancies in the elastic coefficient by construction, and should thus be considered more reliable in the near-contact regime. We will show that the predicted binding energy varies greatly between the different models and should thus be considered unreliable. However, the force vs distance curves for intermediate interlayer distances show significantly less variation. Unlike the energy, the force is not affected by difficulties 
(discussed in Section II C) arising from the mismatch between the large bending stiffness of the cantilever on one hand, and the weakness of the attractive interlayer van der Waals forces on the other hand.

We will finally conclude that the previous theoretical analysis on experimental results ${ }^{19}$ is likely to substantially underestimate the cleavage energy value, but can be used to make reliable predictions of the "peak force" (the minimum force required to cleave the layers). The peak force per unit area is found to be $1.1 \pm 0.15 \mathrm{GPa}$, and occurs $43 \pm 13 \mathrm{pm}$ outside the natural equilibrium interlayer spacing.

\section{THEORY AND ANALYSIS}

As mentioned in the Introduction, we identified a number of problems with the previous theoretical analysis of experimental results ${ }^{19}$. These problems highlight the difficulties of measuring the energetic properties of graphite, a material where direct experiments are difficult and theory is not always comprehensive or conclusive. We outline the identified issues below, with the aim of accounting for each in a reanalysis of the same data.

\section{A. Cleavage vs binding}

We first note that Liu et al. analyzed the energy of cleavage (the energy required to split a bulk along a plane) rather than the bulk-layer binding energy as normally quoted (the energy required to split a bulk into widely separated atomic planes). A discussion of the difference between these energies is found in the supplementary material of Ref. 20, and from a more formal perspective in Ref. 21. While these quantities are usually of the same order of magnitude, they will differ whenever the interaction between second-neighbor planes of atoms is significant, which it typically is in a van der Waals bonded system such as graphene. We will subsequently use $E_{\mathrm{Clv}}$ to refer to the cleavage energy and $E_{\mathrm{BLB}}$ to refer to the bulk-layer binding energy.

While high-level $a b$ initio data is not available for cleavage of graphite, the binding energy of graphite was found by Spanu et al. ${ }^{15}$ with Quantum Monte-Carlo (QMC) method to be $56 \mathrm{meV} /$ Atom $=0.34 \mathrm{~J} / \mathrm{m}^{2}$, and by Lebègue et al. ${ }^{16}$ under the Random Phase Approximation (RPA) to be $48 \mathrm{meV} /$ atom $=0.29 \mathrm{~J} / \mathrm{m}^{2}$. Thrower et $a l .{ }^{17}$ used lower-level theory benchmarked against experimental results for adsorption of polycyclic aromatic hydrocarbons on graphite to predict a graphite binding energy of $57 \pm 4 \mathrm{meV} /$ atom $=0.34 \pm 0.025 \mathrm{~J} / \mathrm{m}^{2}$. The difference between the cleavage and binding energies has also been found on a similar system ${ }^{22}$, and via lower-level theory adjusted to match known high-level theory ${ }^{20}$.

Podeszwa $^{22}$ extrapolated from anthracene, pyrene and coronene to estimate the exfoliation, binding and cleavage energies of graphite. While his model neglects long- ranged (in the plane) plasmon interaction effects due to the finite size of the coronenes, it is expected to yield a good estimate of the relative strengths near contact. He finds a cleavage energy $14 \%$ greater than the binding energy of graphene based on a four sheet model. This gives an absolute cleavage energy of $E_{\mathrm{Clv}}=48.5 \mathrm{meV} / \mathrm{Atom}=$ $0.30 \mathrm{~J} / \mathrm{m}^{2}$. As Podeszwa notes, this extrapolation neglects certain bulk binding properties of graphite, and may underestimate the binding and cleavage energies.

In a recent publication ${ }^{20}$, Björkman et al. investigated bulk properties in various two dimensional materials using various theories. For graphite they found a $2.5 \mathrm{meV} / \mathrm{Ang}^{2}=0.04 \mathrm{~J} / \mathrm{m}^{2}$ difference between the graphite exfoliation and graphene binding energies. Unpublished results ${ }^{23}$ suggest that there is a further $10 \%$ $15 \%$ increase in energy for cleavage compared to exfoliation (an absolute value of $0.03-0.05 \mathrm{~J} / \mathrm{m}^{2}$ based on RPA and QMC results). This is of similar relative magnitude to the energy difference found by Podeszwa ${ }^{22}$, and the energy difference predicted by LJ pair summation models using experimental layer spacing.

These various theories thus predict a cleavage energy $E_{\mathrm{Clv}}$ between $0.30 \mathrm{~J} / \mathrm{m}^{2}$ and $0.39 \mathrm{~J} / \mathrm{m}^{2}$, with a binding energy $E_{\mathrm{BLB}}$ between $0.26 \mathrm{~J} / \mathrm{m}^{2}$ and $0.34 \mathrm{~J} / \mathrm{m}^{2}$ with the lower bound likely underestimated. The cleavage energy $E_{\mathrm{Clv}}=0.19 \pm 0.01 \mathrm{~J} / \mathrm{m}^{2}$ estimated via a theoretical analysis of the cantilever experiment ${ }^{19}$ would correspond to a binding energy of $E_{\mathrm{BLB}}=0.17 \pm 0.01 \mathrm{~J} / \mathrm{m}^{2}$, around half that of the higher-level theories for bulks, and at least $30 \%$ less than the lowest reasonable theoretical prediction.

\section{B. Near-contact force}

Putting aside the distinction between binding and cleavage energies, and taking the model in the previous work ${ }^{19}$ prima facie we note another difficulty that warrants further analysis. The potential model employed is derived directly from the two-parameter LJ potential of Ref. 24. It gives the energy $\Phi_{\mathrm{LJ}}$ as a function of the distance parameter $\nu$ describing the stretching of a single interplanar distance during the cleavage process, while leaving other interplanar distances unchanged. For this paper we rewrite the energy in terms of

$$
x \equiv D-D_{0} \equiv \nu-\nu_{0},
$$

the difference of the distance $D$ between the surfaces after cleavage, and the graphite equilibrium interlayer spacing $D_{0}$ such that $\frac{d}{d x} \Phi_{\mathrm{LJ}}(0)=0\left(\nu_{0}\right.$ is defined as the value of $\nu$ that minimises $\left.\Phi_{\mathrm{LJ}}\right)$.

Equation A6 of Ref. 19's supplementary material gives, after some algebra,

$$
\Phi_{\mathrm{LJ}}(x):=\sum_{n=1}^{\infty} n \Phi_{0}\left(x+\nu_{0}+n \sigma\right),
$$


where

$$
\Phi_{0}(\delta)=\alpha E_{\mathrm{Clv}} \sigma^{2}\left[\frac{2}{5}(\sigma / \delta)^{10}-(\sigma / \delta)^{4}\right]
$$

is the interlayer potential between two layers and is summed over all possible layer interactions to form $\Phi_{\mathrm{LJ}}$. Here $E_{\mathrm{Clv}}$ is the cleavage energy (called the "binding energy" in Ref. 19) while $\alpha$ and $\sigma$ are constants, the latter being related to the interlayer spacing and taking the value $\sigma=0.3415 \mathrm{~nm}$ in the previous analysis. By definition of $x$, the energy takes its minimum value at $x=0$ which gives $\nu_{0}=-0.0045 \mathrm{~nm}$ and $D_{0}=$ $0.337 \mathrm{~nm}$, in agreement with experiment. The constant $\alpha=10.676 \mathrm{~nm}^{-2}$ is chosen to make $\Phi_{\mathrm{LJ}}(0)=-E_{\mathrm{Clv}}$ and can be derived from A2-A6 of the supplementary material for Ref. 19.

The potential $\Phi_{\mathrm{LJ}}$ can be considered a non-linear extension to Hooke's law between planes, reducing to Hooke's law for small displacements. When two graphite surfaces are parallel and near equilibrium lattice spacing, the resulting force should be proportional to the displacement, and should correctly reproduce the $C_{33}$ coefficient of bulk graphite measured in previous experiments ${ }^{25-28}$. In the model (1), small displacements occur when $x \equiv$ $D-D_{0} \approx 0$ such that

$$
\Phi_{\mathrm{LJ}}(x) \approx-E_{\mathrm{Clv}}+\frac{\tilde{C}_{33}}{2 D_{0}} x^{2}, \quad F(x) \approx \tilde{C}_{33} \frac{x}{D_{0}}
$$

where $F(x)$ is the cleavage force near 'contact'. Here

$$
\tilde{C}_{33}=\left.D_{0} \frac{d^{2} \Phi_{\mathrm{LJ}}(x)}{d x^{2}}\right|_{x=0}
$$

is, in fact the elastic coefficient via cleavage, and cannot trivially be compared with existing experiments on bulk graphite. As discussed in Appendix A we can correct (4) to obtain the true $C_{33}$ coefficient for stretching of bulk graphite via

$$
C_{33}=\tilde{C}_{33}+\Delta C_{33},
$$

where $\Delta C_{33}$ is a small correction.

It is thus clear that we can use $\Phi_{\mathrm{LJ}}(x)$ to evaluate the interlayer elastic coefficient $C_{33}$ via (4) and (5). Using $E_{\mathrm{Clv}}=0.19 \mathrm{~J} / \mathrm{m}^{2}$ and $D_{0}=0.337 \mathrm{~nm}$ we find $\tilde{C}_{33}=$ $19.7 \mathrm{GPa}$ and $C_{33}=20.0 \mathrm{GPa}$ (the two coefficients are close enough to be used almost interchangeably), well outside the range of previous experiments ${ }^{25-28}$ which give values between $36.5 \mathrm{GPa}$ and $40.7 \mathrm{GPa}$.

This causes a discrepancy in the overall physical and mechanical model in Ref. 19. For all layers except the open surface, an interplane elastic coefficient of $C_{33}=$ $36.5 \mathrm{GPa}$ [from the stress-strain relationship (A1) presented in the supplementary material] was used. However, at the surface $C_{33}$ is proportional to $E_{\mathrm{Clv}}$ for the potential $\Phi_{\mathrm{LJ}}(x)$ employed, and gives an elastic constant $C_{33}=20.0 \mathrm{GPa}$ [from the interplane potential model $\Phi_{\mathrm{LJ}}(x)$ defined via (1)]. The experimental elastic constant can be matched by setting the cleavage energy to $0.36 \pm 0.02 \mathrm{~J} / \mathrm{m}^{2}$, however this is inconsistent with the value $0.19 \mathrm{~J} / \mathrm{m}^{2}$ deduced by other means in Ref. 19 .
FIG. 1. AFM measurements of one of the cantilevers showing the effect of in-plane stiffness on the profile. Dotted lines are provided as a visual guide. The experimental setup is shown below, showing the scanning direction in (e).
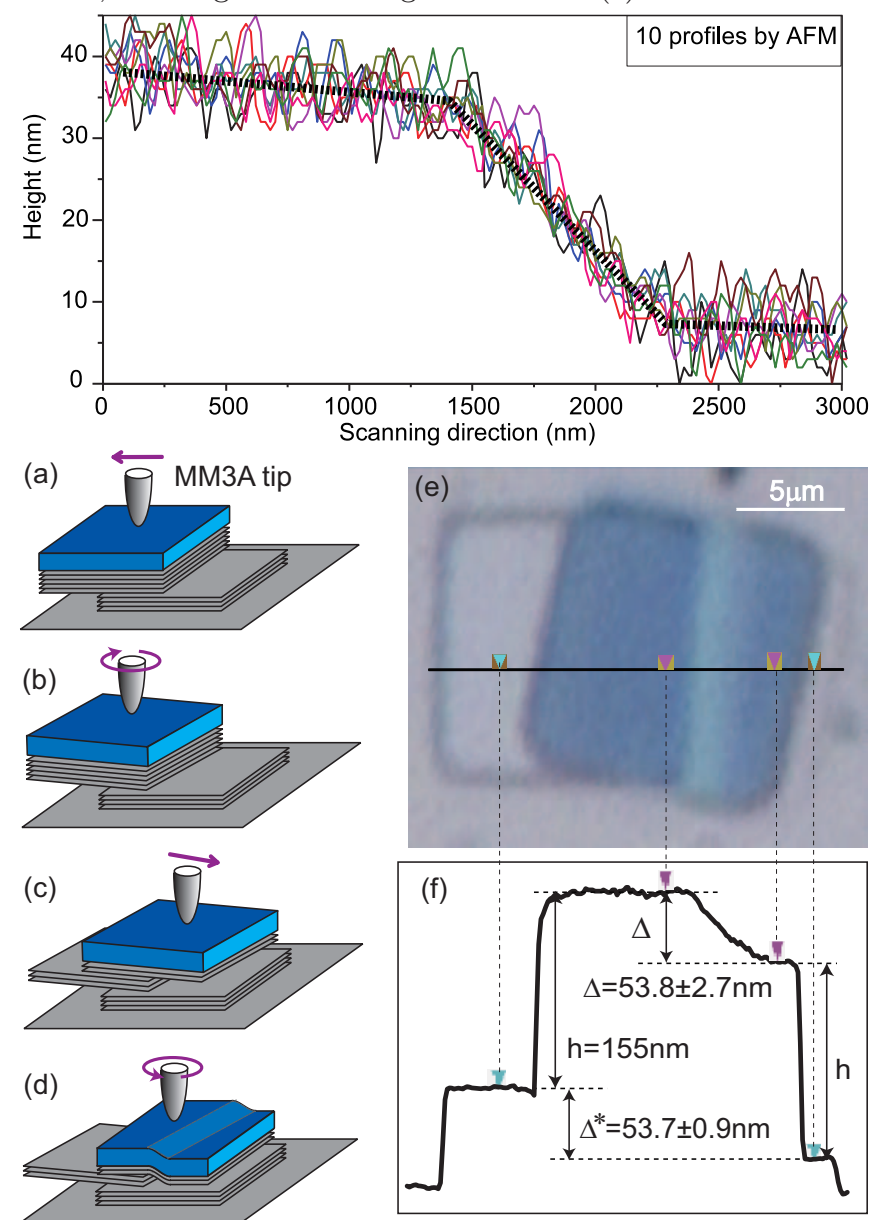

\section{Intermediate and long distance force}

In the experimental setup reported in Ref. 19, graphite was arranged with its layers lying parallel to the base of the cantilevered segment. The bending stiffness of the graphite cantilever is proportional to the in-plane elastic coefficient of graphite (which is one of the largest known at $\sim 1000 \mathrm{GPa}$ ) and the cube of the beam thickness (e.g., $\sim 200 \mathrm{~nm})$. The strong bending stiffness makes bending deformation along the cantilever energetically unfavourable. This stiffness can be seen in the almost straight line of the cantilever profile away from the base and pivot point, as shown here in Figure 1, and in Figure S5 (the FEA illustration) and Figure S7 (the AFM plots) in the same supplementary document. It is clear that the cantilever (bending) deforms minimally until it approaches contact with the base where the forces are sufficient to overcome the internal stiffness.

As such the intermediate part of the binding force will be emphasised in any analysis of the experimental data, while the long distance binding will be harder to deter- 
mine. This makes evaluation of the binding energy difficult as it depends on the integral of the force from infinite separation to finite separation. This also means that the best fit [using (1)] to a given experiment must match both the difficult to measure longer-ranged forces, and the near and intermediate forces. With only one free parameter matching all three ranges is very difficult, and the intermediate region will likely dominate the fit.

The experimental difficulties are further amplified by the fact that theoretical understanding of the longerranged van der Waals potential of graphite cleavage is limited. Here high-level theory results ${ }^{29}$ are valid only for the extreme outer limit where corrections for finite Dirac cones ${ }^{30}$ and other non-asymptotic physics are not required.

This lack of theoretical insight comes from difficulties in understanding the contribution of the $\pi_{z}$ and $s p_{2}$ orbitals on the dispersion processes involved in cleavage. While these dispersion forces contribute a signficant amount to the intermediate region energy profile $(\sim 45 \%$ of the energy at contact for graphite, bigraphene and exfoliation ${ }^{31}$ ), they contribute comparitively less to the intermediate force. Thus a failure to properly account for dispersion is less problematic for interpretation of peak force measurements compared to the cleavage energy.

Furthermore, the dispersion contribution is itself split into two components, which dominate at different length scales. Here the $s p_{2}$ orbitals dominate the dispersion in the intermediate region, while $\pi_{z}$ orbitals dominate in the asymptotic region. The different force and length scales involved in dispersion thus make extrapolation from the near-contact region to the asymptotic region very difficult. This makes mathematical fitting of an all-region force unreliable without firm theoretical backing, with consequences for the energy. It does not, however, affect measurement of near-contact forces, which depend minimally on the unknown asymptotics.

\section{ALTERNATE MODELS}

As mentioned in Section II B, the Lennard-Jones model (1) with cleavage energy determined by best fit to experiment failed to reproduce the known near-contact force of graphite. Ideally any model potential should reproduce the correct near-contact force by construction, via the experimentally measured interlayer distance $D_{0}=0.334 \mathrm{~nm}$, and the experimental elastic coefficient $C_{33}=36.5 \mathrm{GPa}$. Additionally the potential should decay ${ }^{29}$ as the van der Waals power law $-C_{2} D^{-2}$ well away from contact. A model potential can then be formed that allows the binding energy to vary as a parameter, while matching these known properties of graphite. The LJ potential, with just two parameters, is not able to simultaneously fit $D_{0}, C_{33}$ and $E_{\mathrm{Clv}}$, and leads to $C_{33} \propto E_{\mathrm{Clv}}$. We thus propose two new models of the binding energy that decouple the elastic coefficient from the cleavage energy, and thus allow better reproduction of known properties of graphite.

Our first model is designed to reproduce the RPA energy curve in the near-contact region when the input energy takes the RPA value $E_{\mathrm{Clv}}=0.29 \mathrm{~J} / \mathrm{m}^{2}$. Here, in addition to matching the binding distance and elastic coefficient, we match the non-linear coefficient $C_{333}=$ $-540 \mathrm{GPa}$ obtained from the RPA so that for small $x / D_{0}$ the force obeys $F(x) \approx C_{33}\left(x / D_{0}\right)+\frac{1}{2} C_{333}\left(x / D_{0}\right)^{2}$. One such "near-contact model" (NCM) is

$$
\Phi_{\mathrm{NCM}}(x)=\frac{-E_{\mathrm{Clv}}}{1+c_{2} x^{2}+c_{3} x^{3} e^{-k x}}
$$

where $x=D-D_{0}$ is the deviation from the experimental lattice spacing. Here $c_{2}=C_{33} /\left(2 D_{0} E_{\mathrm{Clv}}\right)$ and $c_{3}=C_{333} /\left(6 D_{0}^{2} E_{\mathrm{Clv}}\right)$ ensure that the first three derivatives of $\Phi_{\mathrm{NCM}}$ are equal to their RPA values at contact $D=D_{0}$ for arbitrary $E_{\mathrm{Clv}}$. Free parameter $k>0$ guarantees that $\Phi_{\mathrm{NCM}}(x \rightarrow \infty) \propto x^{-2}$. We choose $k=8$ to ensure that the force $\frac{\mathrm{d} \Phi_{\mathrm{NCM}}}{\mathrm{d} x}$ is single peaked for $x>0$, and that the RPA data is approximately reproduced when the RPA energy $E_{\mathrm{Clv}}=0.29 \mathrm{~J} / \mathrm{m}^{2}$ is used.

Alternatively, one may wish to keep the LJ-like form of the model potential, while reproducing the known physical properties $D_{0}$ and $C_{33}$ as well as the correct $-C_{2} / D^{2}$ asymptotic form (if not its coefficient $C_{2}$ ). This suggests a model potential of general form

$$
\Phi_{\mathrm{LJ}(\mathrm{p})}(x)=\frac{E_{\mathrm{Clv}}}{p-2}\left[\frac{2}{\left(1+x / D_{0}\right)^{p}}-\frac{p}{\left(1+x / D_{0}\right)^{2}}\right]
$$

where the exponent $p=C_{33} D_{0} /\left(2 E_{\mathrm{Clv}}\right)$ is chosen to ensure the experimental $C_{33}$ elastic coefficient can be reproduced. Such an approach is perhaps physically less justified than the above in the near contact region, but does not require an RPA third-order elastic coefficient as additional input, and may be more accurate away from contact.

\section{RESULTS}

Following the previous work ${ }^{19}$, we use the three different model potentials $\Phi_{\mathrm{LJ}}, \Phi_{\mathrm{NCM}}$ and $\Phi_{\mathrm{LJ}(\mathrm{p})}$ [from respectively equations (1), (6) and (7)] in FEA models to determine a model-dependent cleavage energy $E_{\mathrm{Clv}}$. This was defined to be the value (for each model) that caused the elastically deformed curves determined via FEA to best match the experimentally measured profiles. The variation of the predicted binding energy was very great even within a given example geometry, ranging from $E_{\mathrm{Clv}}=0.13 \mathrm{~J} / \mathrm{m}^{2}$ for the near-contact model $\Phi_{\mathrm{NCM}}$, to $E_{\mathrm{Clv}}=0.19 \mathrm{~J} / \mathrm{m}^{2}$ for the LJ model $\Phi$ and $E_{\mathrm{Clv}}=0.27 \mathrm{~J} / \mathrm{m}^{2}$ for the modified LJ model $\Phi_{\mathrm{LJ}(\mathrm{p})}$. The modified LJ model best matches previous theoretical predictions of the cleavage energy, but the error bar across the three models is too signficant for any result to be considered reliable. This is unsurprising as the cleavage energy depends partly on the very weak dispersive forces at 
FIG. 2. Cleavage force per unit area under the three different models plotted against the inter-layer distance $D$ (where the equilibrium interlayer spacing is $D_{0}=0.334 \mathrm{~nm}$ ). The RPA bulk layer binding force is included for illustrative purposes, although it is not in general the same as the cleavage force.

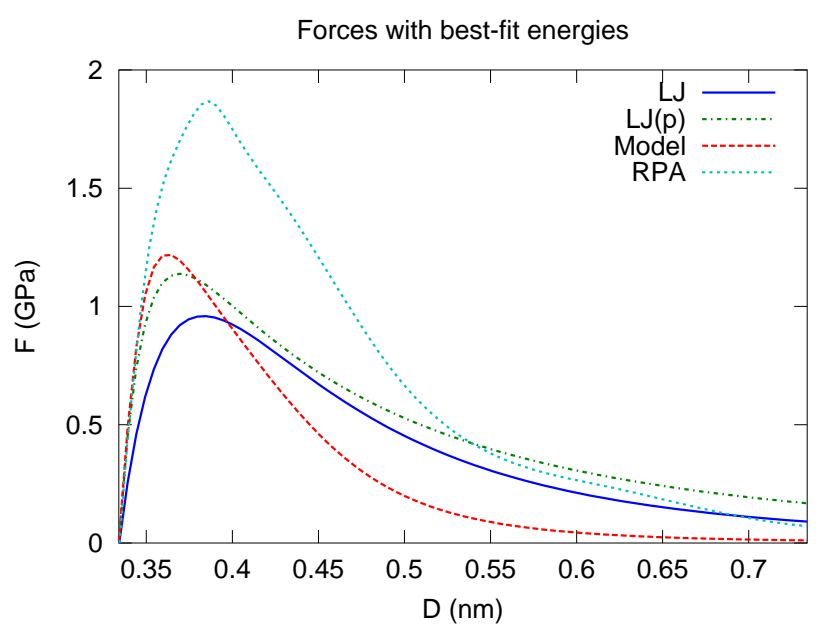

TABLE I. Best fit cleavage energy, and corresponding peak force and position for different models. RPA results are provided for illustration, not comparison.

\begin{tabular}{lrrr}
\hline \hline Model & $E_{\mathrm{Clv}}\left(\mathrm{J} / \mathrm{m}^{2}\right)$ & $F_{m}(\mathrm{GPa})$ & $D_{m}(\mathrm{~nm})$ \\
\hline $\mathrm{LJ} \Phi$ & 0.19 & 0.96 & 0.388 \\
$\mathrm{NCM} \Phi_{\mathrm{NCM}}$ & 0.13 & 1.22 & 0.367 \\
$\mathrm{LJ}(p) \Phi_{\mathrm{LJ}(\mathrm{p})}$ & 0.27 & 1.13 & 0.372 \\
\hline RPA Binding ${ }^{a}$ & 0.29 & 1.87 & 0.385 \\
\hline \hline
\end{tabular}

large distance, which are difficult to determine accurately via the current experimental setup ${ }^{19}$. Additionally, the asymptotic van der Waals behaviour for cleavage is unknown, and none of the models can be guaranteed to hold true in the more distant limit.

From the potential it is trivial to determine the force $F=\frac{\mathrm{d} \Phi}{\mathrm{d} x}$, and in the near-contact region this should be less model-dependent and given more reliably by experiment. Indeed by construction both $\Phi_{\mathrm{NCM}}$ and $\Phi_{\mathrm{LJ}(\mathrm{p})}$ will predict identical linearly varying forces near the inter-layer binding distance. Using the best fit $E_{\mathrm{Clv}}$ parameters for each model yields distance-dependent forces displayed in Figure 2. It is immediately apparent that while the force in the tail varies greatly between the models, the position and magnitude of the "peak" force (the maximum) varies much less signficantly. The "peak force" is a physically important property of graphite, giving the force per unit area require to "cleave" graphite in two at a single surface. This agrees with the arguments given in Section II C that the analysis of experiment of Ref. 19 will be most sensitive to the force in the intermediate range.

Using the best fit $E_{\mathrm{Clv}}$ for each of the three models, we semi-analytically determine the peak force $F_{m}$ and the inter-layer distance $D_{m}$ at which it occurs. We tabulate the results in Table I, including the peak bulk layer binding (as opposed to cleavage) force predicted by the RPA for illustrative comparison. The peak force varies by up to $27 \%$ over all three models, which is very good agreement for such different models. For the two models $\Phi_{\mathrm{NCM}}$ and $\Phi_{\mathrm{LJ}(\mathrm{p})}$ with the experimental elastic coefficient of bulk graphite included as a parameter the variation is even smaller still, with only a $5 \mathrm{pm}$ difference to the position and an $8 \%$ difference in the peak force. This small variation is a very positive feature, as the cleavage energy parameter varies by over $100 \%$.

The peak force predicted by the RPA is significantly larger than those found via the analysis of the experiment with any of the models. While at first glance this is worrying, we note that the RPA data is for the different problem of bulk layer binding (under uniaxial stretching perpendicular to the planes) and cannot be directly compared. Indeed one expects the force laws for cleavage, exfoliation and bulk layer binding to show greater variation than the 'binding' energies as each obeys a different asymptotic power law ${ }^{29}$, with bulk Coulomb screening reducing the dispersion force in cleavage and exfoliation. This screening effect suggests that the peak force of cleavage should be less than that of bulk layer binding, and thus one should expect an apparent discrepancy between i) the values of peak forces deduced from the experiments using the three models, and ii) theoretical predictions from the RPA. Full resolution of the differences between peak force in bulk-layer binding and cleavage would require further experiments and/or further RPA calculations (or other high-level theory) for the cleavage geometry. These are beyond current capabilities, however.

\section{v. CONCLUSION}

Overall we conclude that the binding energy evaluated through the theoretical analysis of experimental results in the previous work (Ref. 19) is likely to be substantially underestimated. Firstly, the model employed in Ref. 19 yields a near-contact force law with an elastic coefficient of $C_{33}=20.0 \mathrm{GPa}$, just over half that of previous reliable experiments ${ }^{25-28}$ with $C_{33}=38.6 \pm 2.1 \mathrm{GPa}$. The employed LJ model makes $C_{33} \propto E_{\mathrm{Clv}}$ suggesting that $E_{\mathrm{Clv}} \approx 0.36 \mathrm{~J} / \mathrm{m}^{2}$ would be required to match the experiment elastic parameter. Secondly, the experimental setup and model potential [see our (1) or (A6) of the supplementary material of Ref. 19] are designed for the measurement of cleavage energies $E_{\mathrm{Clv}}$, not the bulk-layer binding energies $E_{\mathrm{BLB}}$ with which their results were compared. It is believed that $E_{\mathrm{BLB}} \approx 0.85 E_{\mathrm{Clv}}$ in graphitic systems, leading to a value $E_{\mathrm{BLB}} \approx 0.17 \mathrm{~J} / \mathrm{m}^{2}$ from the experimental analysis, approximately half the value estimated by high-level theory ${ }^{15,16,20,22}$.

By attempting new fits to the same data, using model potentials which include the experimental $C_{33}$ coefficient as an input, we find that the predicted cleavage en- 
ergy $E_{\mathrm{Clv}}$ varies dramatically (by 100\%) depending on the model potential used. However, the size and position of the peak force deduced from the experiment (the minimum force per unit area required to fully "cleave" graphite at a surface) is much less dependent on the model, showing only a $27 \%$ variation in magnitude across all three models, reduced to $8 \%$ between the two models that reproduce the experimental $C_{33}$ coefficient. We thus conclude that the peak force per unit area can be accurately predicted by this experiment, and that it is $1.1 \pm 0.15 \mathrm{GPa}$ and occurs at an inter-layer spacing of $0.377 \pm 0.013 \mathrm{~nm}$, where the error bar includes predicted experimental and analysis errors, as well as variation across the three interaction models.

The present experimental arrangement, and lack of asymptotic van der Waals theory for cleavage makes it difficult to extract accurate cleavage energies. However, similar experiments (e.g., a graphite flake cantilever with fewer graphene layers), in principle, can be devised that allow accurate measurement of the force over a wider range of distances. Such experiments would enable the cleavage energy to be determined experimentally by integration over the force, and these will be the focus of future efforts. Similarly it would be desirable to better understand at the theoretical level the asymptotic van der Waals attractive potential in graphite under cleavage, and this is also a topic for future investigation.

Finally, we note that these difficulties in resolving asymptotic properties (and thus integrated quantities like the binding energy) are likely to occur in analysis of indirect force measurements for all layered materials, not just graphite. Although they are expected to be most severe in graphite due to the contribution of the $\pi_{z}$ dispersion. We thus recommend against the use of the Lennard-Jones models for layered systems, and recommend instead the use of something like the $\mathrm{LJ}(p)$ model (7) when modelling interlayer forces in systems with known elastic coefficients.

\section{ACKNOWLEDGMENTS}

We would like to thank Torbjörn Björkman for helpful discussion on cleavage, exfoliation and binding in layered solids. T.G. and J.F.D. were supported by ARC Discovery Grant DP1096240. S.L. acknowledges financial support from the Université de Lorraine through the program "Soutien à la dimension internationale de la recherche". Q.S.Z. acknowledges the financial support from NSFC through Grants No. 10832005 and No. 10972113, the 973 Program through Grants No. 2007CB936803 and No. 2013CB934200. J.Z.L. acknowledges Seed Grant 2013 from the engineering faculty of Monash University.

\section{Appendix A: Elastic coefficients}

In general the $C_{33}$ elastic coefficient is the ratio of interlayer force to interlayer displacement induced by stretching all layers evenly (with appropriate scaling). By contrast the model used in Ref. 19 involves displacement between two particular layers (cleavage) with other layer spacing kept essentially fixed, a process associated with elastic constant $\tilde{C}_{33}$. Employing the interlayer potential $\Phi_{0}$ [equation (2)] we can find the coefficient in the two different models via [using $x=\nu-\nu_{0}$ in equation (4) for $\tilde{C}_{33}$ and a similar derivation for $C_{33}$ ]

$$
\begin{aligned}
& \tilde{C}_{33}=\left.D_{0} \frac{d^{2}}{d \nu^{2}} \sum_{n=1}^{\infty} n \Phi_{0}(n \sigma+\nu)\right|_{\nu=\nu_{0}}, \\
& C_{33}=\left.D_{0} \frac{d^{2}}{d \nu^{2}} \sum_{n=1}^{\infty} \Phi_{0}(n \sigma+n \nu)\right|_{\nu=\nu_{0}},
\end{aligned}
$$

where (A2) should reproduce the true $C_{33}$ coefficient of graphite for a good interlayer potential model. The difference between these two coefficients is

$$
\begin{aligned}
\Delta C_{33} & \equiv C_{33}-\tilde{C}_{33} \\
& =D_{0} \sum_{n=2}^{\infty} \frac{d^{2}}{d \nu^{2}}\left[\Phi_{0}(n \sigma+n \nu)-n \Phi_{0}(n \sigma+\nu)\right]_{\nu=\nu_{0}}
\end{aligned}
$$

since the $n=1$ terms cancel. We thus expect $\Delta C_{33}$ to be small compared to $C_{33}$ as $\Phi_{0}(2 \sigma) \ll \Phi_{0}(\sigma)$ and $\left|\nu_{0}\right| \ll$ $\sigma$. Inserting the experimental parameters $\nu_{0} / \sigma=-0.13$, $D_{0}=0.337 \mathrm{~nm}$ and $E_{\mathrm{BLB}}=0.19 \mathrm{~J} / \mathrm{m}^{2}$ gives $\Delta C_{33}=$ $0.26 \mathrm{GPa}$ and $\tilde{C}_{33}=19.7 \mathrm{GPa}$. As expected the correction is small at around $1.3 \%$.

${ }^{1}$ K. Novoselov, A. Geim, S. Morozov, D. Jiang, Y. Zhang, S. Dubonos, I. Grigorieva, and A. Firsov, Science 306, 666 (2004).

2 A. K. Geim and K. S. Novoselov, Nature Materials 6, 183 (2007).

${ }^{3}$ M. I. Katsnelson, Materials Today 10, 20 (2006).

${ }^{4}$ A. H. Castro Neto, F. Guinea, N. M. R. Peres, K. S. Novoselov, and A. K. Geim, Rev. Mod. Phys. 81, 109 (2009).

${ }^{5}$ M. I. Katsnelson, K. S. Novoselov, and A. K. Geim, Nature Physics 2, 620 (2006).

${ }^{6}$ L. Hu and G. Gruner, U.S. Patent No. 8390589 B2 (5 Mar 2013).

${ }^{7}$ K. S. Kim, Y. Zhao, H. Jang, S. Y. Lee, J. M. Kim, K. S. Kim, J. H. Ahn, P. Kim, J. Choi, and B. H. Hong, Nature 457, 706 (2009).

${ }^{8}$ L. A. Girifalco and R. A. Lad, The Journal of Chemical Physics 25, 693 (1956).

${ }^{9}$ L. X. Benedict, N. G. Chopra, M. L. Cohen, A. Zettl, S. G. Louie, and V. H. Crespi, Chem. Phys. Letters 286, 490 (1998).

${ }^{10}$ R. Zacharia, H. Ulbricht, and T. Hertel, Phys. Rev. B 69, 155406 (2004).

${ }^{11}$ M. Dion, H. Rydberg, E. Schröder, D. C. Langreth, and B. I. Lundqvist, Phys. Rev. Lett. 92, 246401 (2004).

${ }^{12}$ S. D. Chakarova-Käck, E. Schröder, B. I. Lundqvist, and D. C. Langreth, Phys. Rev. Lett. 96, 146107 (2006).

${ }^{13}$ E. Ziambaras, J. Kleis, E. Schröder, and P. Hyldgaard, Phys. Rev. B 76, 155425 (2007).

${ }^{14}$ M. Hasegawa and K. Nishidate, Phys. Rev. B 70, 205431 (2004).

${ }^{15}$ L. Spanu, S. Sorella, and G. Galli, Phys. Rev. Lett. 103, 196401 (2009). 
${ }^{16}$ S. Lebègue, J. Harl, T. Gould, J. G. Ángyán, G. Kresse, and J. F. Dobson, Phys. Rev. Lett. 105, 196401 (2010).

${ }^{17}$ J. D. Thrower, E. E. Friis, A. L. Skov, L. Nilsson, M. Andersen, L. Ferrighi, B. Jrgensen, S. Baouche, R. Balog, B. Hammer, and L. Hornekr, The Journal of Physical Chemistry C 117, 13520 (2013).

${ }^{18}$ T. c. v. Bučko, S. Lebègue, J. Hafner, and J. G. Ángyán, Phys. Rev. B 87, 064110 (2013).

${ }^{19}$ Z. Liu, J. Z. Liu, Y. Cheng, Z. Li, L. Wang, and Q. Zheng, Phys. Rev. B 85, 205418 (2012).

${ }^{20}$ T. Björkman, A. Gulans, A. V. Krasheninnikov, and R. M. Nieminen, Phys. Rev. Lett. 108, 235502 (2012).

${ }^{21}$ T. Gould, K. Simpkins, and J. F. Dobson, Phys. Rev. B 77, 165134 (2008).

${ }^{22}$ R. Podeszwa, The Journal of Chemical Physics 132, 044704 (2010).
${ }^{23}$ T. Björkman and A. Gulans, (private communication).

${ }^{24}$ L. A. Girifalco and M. Hodak, Phys. Rev. B 65, 125404 (2002).

${ }^{25}$ O. L. Blakslee, D. G. Proctor, E. J. Seldin, G. B. Spence, and T. Weng, Journal of Applied Physics 41, 3373 (1970).

${ }^{26}$ W. B. Gauster and I. J. Fritz, Journal of Applied Physics 45, 3309 (1974).

${ }^{27}$ N. Wada, R. Clarke, and S. Solin, Solid State Communications 35, 675 (1980).

${ }^{28}$ A. Bosak, M. Krisch, M. Mohr, J. Maultzsch, and C. Thomsen, Phys. Rev. B 75, 153408 (2007).

${ }^{29}$ T. Gould, E. Gray, and J. F. Dobson, Phys. Rev. B 79, 113402 (2009).

${ }^{30}$ T. Gould, J. F. Dobson, and S. Lebègue, Phys. Rev. B 87, 165422 (2013).

${ }^{31}$ T. Gould, S. Lebègue, and J. F. Dobson, Journal of Physics: Condensed Matter 25, 445010 (2013). 